\title{
Deep Transfer Learning Application for Automated Ischemic Classification in Posterior Fossa CT Images
}

\author{
Anis Azwani Muhd Suberi ${ }^{1}$, Wan Nurshazwani Wan Zakaria ${ }^{*}$, , Razali Tomari ${ }^{3}$ \\ Ain Nazari ${ }^{4}$, Mohd Norzali Hj Mohd ${ }^{5}$, Nik Farhan Nik Fuad ${ }^{6}$ \\ Faculty of Electrical and Electronic Engineering (FKEE) $1,2,3,4,5$ \\ Universiti Tun Hussein Onn Malaysia (UTHM) \\ 86400 Parit Raja, Batu Pahat, Johor, Malaysia \\ UKM Medical Centre, Jalan Yaacob Latif ${ }^{6}$ \\ Bandar Tun Razak, 56000 Cheras \\ Kuala Lumpur, Malaysia ${ }^{6}$
}

\begin{abstract}
Computed Tomography (CT) imaging is one of the conventional tools used to diagnose ischemic in Posterior Fossa (PF). Radiologist commonly diagnoses ischemic in PF through CT imaging manually. However, such a procedure could be strenuous and time consuming for large scale images, depending on the expertise and ischemic visibility. With the rapid development of computer technology, automatic image classification based on Machine Learning (ML) is widely been developed as a second opinion to the ischemic diagnosis. The practical performance of ML is challenged by the emergence of deep learning applications in healthcare. In this study, we evaluate the performance of deep transfer learning models of Convolutional Neural Network (CNN); VGG-16, GoogleNet and ResNet-50 to classify the normal and abnormal (ischemic) brain CT images of PF. This is the first study that intensively studies the application of deep transfer learning for automated ischemic classification in the posterior part of brain CT images. The experimental results show that ResNet-50 is capable to achieve the highest accuracy performance in comparison to other proposed models. Overall, this automatic classification provides a convenient and time-saving tool for improving medical diagnosis.
\end{abstract}

Keywords-Deep learning; ischemic stroke; posterior fossa; classification; convolutional neural network; computed tomography; medical diagnosis

\section{INTRODUCTION}

Ischemic stroke is a condition where there is a blood clot in the blood vessel which causes a blockage to the area of the brain [1]-[3]. Recent studies have shown that ischemic stroke is the third leading causes of mortality across the world, accounting for about $74.8 \%$ of the patient are experiencing their first episode of stroke in general [4]. The mortality rate of ischemic is also increasing with 3 million deaths globally [5]. There have been tons of ischemic are missed at the early stage of diagnosis which requires an accurate diagnosis to defeat the mortality rate. The delay of early diagnosis and treatment would stimulate the growth of acute ischemic which then reducing the chances rate of the patient to survive [6]. To date, Computed Tomography (CT) is the most applicable tool for rapid screening of ischemic in medical emergency [3][6][7]. Despite that, the diagnosis of ischemic in CT slices of Posterior Fossa (PF) remains as the most challenging area, which demands the expertise of radiologist [8]-[10]. The diagnosis process can be monotonous and time-consuming. Generally, the human brain consists of PF which covers two major components; Cerebellum and brainstem. The cerebellum is the primary site for body coordination and movement while the brainstem has been implicated for respiration. The beam hardening artifacts produced by thick bone and inadequate contrast resolution usually limits the performance of CT in PF slices [9]. False positive cases usually occur because of the behavioural nature of acute ischemic in loss of gray-white matter differentiation which nearly similar to normal tissues. The sensitivity of CT in the first 24 hours of ischemic inspection in PF slices achieves a low performance with only $41.8 \%$ [10]. Fig. 1 shows the example of ischemic case in PF region.

Computer Aided Diagnosis (CAD) has been widely developed in the medical image analysis to provide support for the radiologist in the decision-making process [11]-[14]. There are various techniques applied in the CAD to classify normal and ischemic CT slices using traditional Machine Learning (ML) based method. Typically, these CAD systems are starting with the pre-processing, followed by hand-crafted feature extraction and finally the classification steps to discriminate normal and abnormal slices [11][14][15]. Texture, intensity features and wavelet transform are the examples of features which have been extracted to be fed into Support Vector Machine (SVM), Artificial Neural Network (ANN) and KNearest Neighbour (KNN) as the input [11][14]-[16].

At present, Deep Learning (DL) has made a breakthrough in medical image classification owing to its superior performance in solving a wide range of problems [17]. Contrary to the traditional ML methods, DL is capable to directly learn the features of an image without a hand-crafted feature extraction step. The emergence of DL in medical image classification with diseases such as Alzheimer, brain tumour ischemic infarction and dementia are tremendously increasing with high performance [18]. However, only very recently have other researchers started to investigate the possibility of ischemic image classification with acute condition based on the DL method [12][19][20]. To the best of author knowledge, this is the first study to explore the challenge of classifying normal and ischemic primarily in PF slices using DL.

*Corresponding Authors 


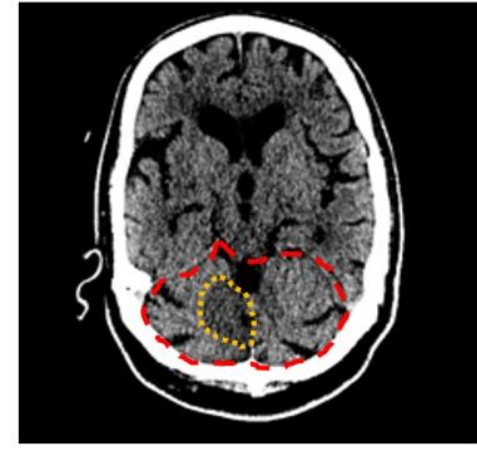

PF region - - -

Ischemic

Fig. 1. Ischemic Stroke in PF.

Generally, the huge amount of CT image data affect the computational cost to train the DL network [21]. Convolutional Neural Network (CNN) is the most popular DL architecture [21][22]. Through the ImageNet Large Scale Visual Recognition Challenge (ILSVRC), various pre-trained models of CNN have been developed with high boosting performance [23]-[25]. Pre-trained models can be another way of overcoming the problems of training $\mathrm{CNN}$ with huge data since the Graphics Processing Unit (GPU) hardware demands a highcost. This is because the previous pre-trained models are able to apply the knowledge of transfer learning to another domain. Those models are only required to train the last dense layer since they use the similar weight of convolutional layers from the previous pre-trained.

There are several attempts which can be enumerated for deciding a patient with an acute ischemic stroke. These are usually intensive and lack of robustness due to the unknown stroke symptom onset [26]. Therefore, Pereira et al. [19] employ a CNN classifier for brain ischemic CT images by combining with Particle Swarm Optimization (PSO). They use CIFAR-10 and ImageNet architecture as a deep convolutional network model for classification. Chin et al. [20] develop a CNN network to classify ischemic using image patches. They use two convolutional layers, one max pooling layer and a single fully connected layer. More recently, Dourado et al. [12] develop a classifier for ischemic and haemorrhage stroke by combining ML-based classifier with CNN. They use the CNN as feature extractor instead of the hand-crafted feature extraction.

Despite all efforts, no specific study exist today that can provide a classification of normal and ischemic in PF slice. It is important to note that only $20 \%$ of ischemic stroke is represented by posterior [27]. This eventually influences the vast amount of training data needed for DL. Thus, this study employs and evaluates the pre-trained models as deep transfer learning in CNN towards classifying normal and ischemic in PF slices of CT. Early diagnosis of ischemic in PF is extremely important to prevent the brainstem infarction incidence which can impact the prolonged state of the patient [10]. The significant contributions of this study can be listed as follows to address this unaccounted phenomenon: (a) comparative study of pre-trained models to classify normal and ischemic mainly in PF slices of CT, and (b) presentation of cross validation to improve the performance of the predictive models. The following paper is organised as follows:

This study is supported by Universiti Tun Hussein Onn Malaysia and UKM Medical Centre under TIER 1 Vot H203 and GPPS Vot U954 Research (a) Section 2 describes the state-of-the-art of CNN models; Section 3 presents the research method used including brain dataset and research workflow; Section 4 shows the experimental results for initial training and $k$-fold cross validation, followed by Section 5 which summarises the findings.

\section{DEEP TRANSFER LEARNING MODEL OF CNN}

The pre-trained network is shown to operate effectively in the DL field. This is particularly important for small training dataset cases. The pre-trained models appear to possess relatively good performance with previous knowledge of large scale data of the existing model. This section describes a background on three popular pre-trained models (VGG-16, GoogleNet and ResNet-50) which have been proven to be excellent in medical image classification [28][29].

\section{A. $V G G-16$}

VGG-16 is the CNN model developed by Simonyan and Zisserman [25] with the best performance of $92.7 \%$ top-5 test accuracy in ImageNet. Fig. 2 illustrates the architecture model of VGG-16. This was developed and tested on a number of 1000 classes. The input required for the convolution layer is RGB with a fixed size of $224 \times 224$. The input image is passed via a stack of convolutional layers with ReLU activations in which filters are applied with very small receptive fields of $3 \times 3$. Meanwhile, the stride of convolution is fixed to 1 . In the context of spatial pooling, five max-pooling layers are performed after the convolutional layers. Each of the maxpooling is typically used with a window of $2 \times 2$ pixels and a stride of 2. The stack of convolutional layers is followed by three fully connected (fc) layers and finally, the softmax layer. The ability of VGG to perform through an increase of effective network receptive field is one of the major advantages due to stacking stage of multiple convolution layers with small kernels as well as limiting the number of parameters [28]. For a number of years, this model in literature has been prevalent to the area of medical image classification such as mammograms [30].

\section{B. GoogleNet}

GoogLeNet is developed by Szegedy et al. [24] which delivers the best performance in ILSVRC 2014 with an error rate of $6.67 \%$. The model is implemented using the strategic approach of stacking nine Inception modules. The implementation presents the advantage of employing optimal local sparse structure using dense components of a multi-layer network [28]. Particularly, the Inception module has included four branches. The first branch applies $1 \times 1$ convolution to perform a linear transformation to each input channel. Meanwhile, the second and third branches of GoogleNet exploit the principle of including $1 \times 1$ kernel convolution with kernel sizes of $3 \times 3$ and $5 \times 5$, respectively. The idea behind this is to perform a dimensionality reduction before applying maxpooling and convolution with $1 \times 1$ kernel in the fourth branch. Meanwhile, the purpose of using max-pooling is to reduce the feature maps dimensionality. The final stage of the architecture highlights the implementation of average pooling layer, fc layer, drop out and softmax loss as the classifier. The details of these structures are depicted in Fig. 3. 


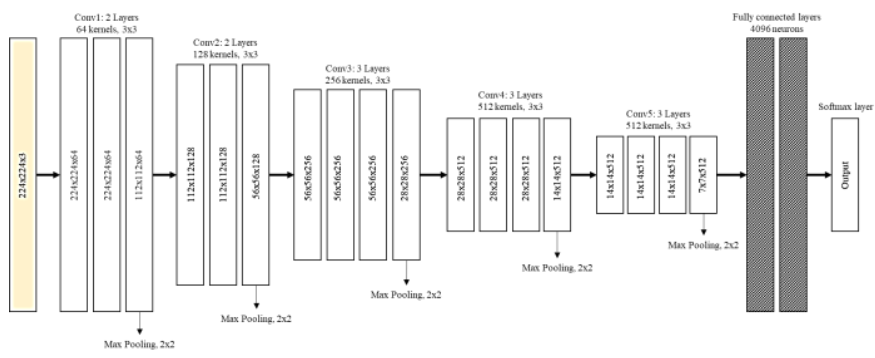

Fig. 2. VGG-16 Architecture [28].

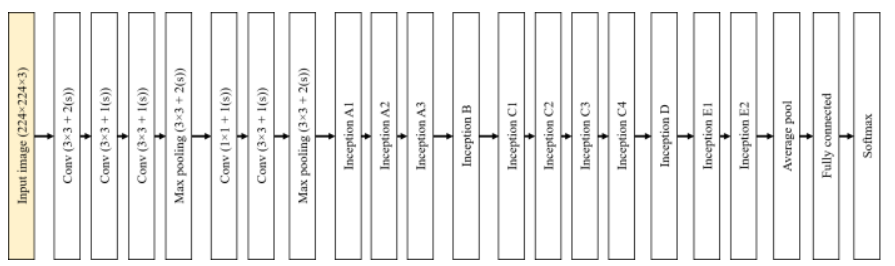

Fig. 3. GoogleNet Architecture [31].

\section{ResNet-50}

ResNet-50 or Residual Network is introduced by He et al. [23] in ILSVRC 2015. Similar with VGG-16 and GoogleNet, ResNet-50 also has been trained on the ImageNet database of 1000 categories with the superior performance of $3.57 \%$ error rate. ResNet-50 architecture is composed of convolutional layers, pooling layers and multiple residual layers with each consisting residual blocks of convolutional layers and batch normalization layers [30]. The configuration of ResNet-50 particularly consist of four residual layers, a dense layer and followed by the output layer with softmax activation function [30]. The design and implementation of each convolutional layer in a residual block are added with shortcut connection before propagating the output to the subsequent block as shown in Fig. 4. ResNet-50 provides an advantage in delivering a remarkable speed-up convergence as compared to other pretrained models [18].

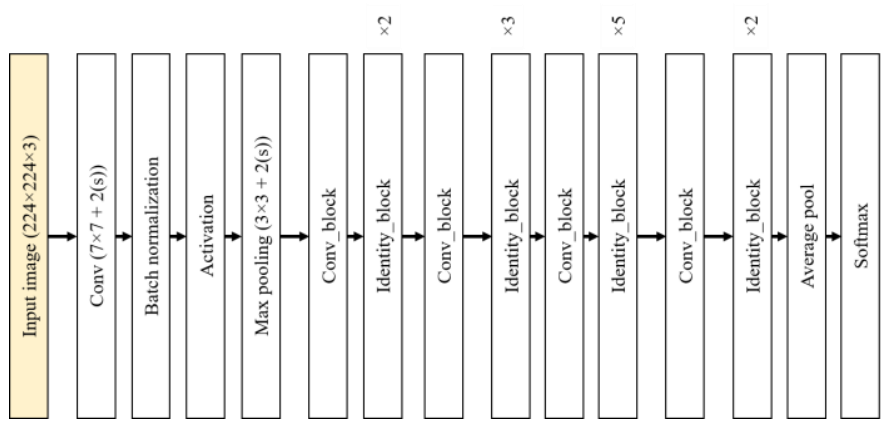

Fig. 4. ResNet-50 Architecture [31].

\section{RESEARCH METHOD}

As aforementioned, the main contribution of this work is to evaluate the performance of deep transfer learning under the context of ischemic classification in PF of the CT images. Fig. 5 illustrates the proposed workflow which the training and validation images undergo 1) Digital Imaging and Communications in Medicine (DICOM) image conversion, and 2) Data augmentation and 3) CNN pre-trained model. The deep transfer learning of $\mathrm{CNN}$ model has been implemented with
MATLAB R2018B software. All experiments are performed on Intel ${ }^{\circledR}$ Core $^{\mathrm{TM}}$ I7-7500U processor with $2.90 \mathrm{GHz}$ and $8 \mathrm{~GB}$ RAM platform.

\section{A. Brain Dataset}

Brain CT images are acquired from the UKM Medical Centre. The images are scanned using Aquilion One by Toshiba scanner. The dataset contains normal and abnormal images. The abnormal images belong to acute ischemic in PF region. Only images with $\mathrm{PF}$ are used from the database except for non-PF slices. The size of all images is $512 \times 512$ pixels in an axial plane. 400 of images are used for training and validation. Among these images, 200 contains ischemic in PF and the rest of 200 are normal images. Fig. 6 shows a few sample CT images of the brain dataset. The ischemic is clinically confirmed and marked (green region) by an experienced radiologist.
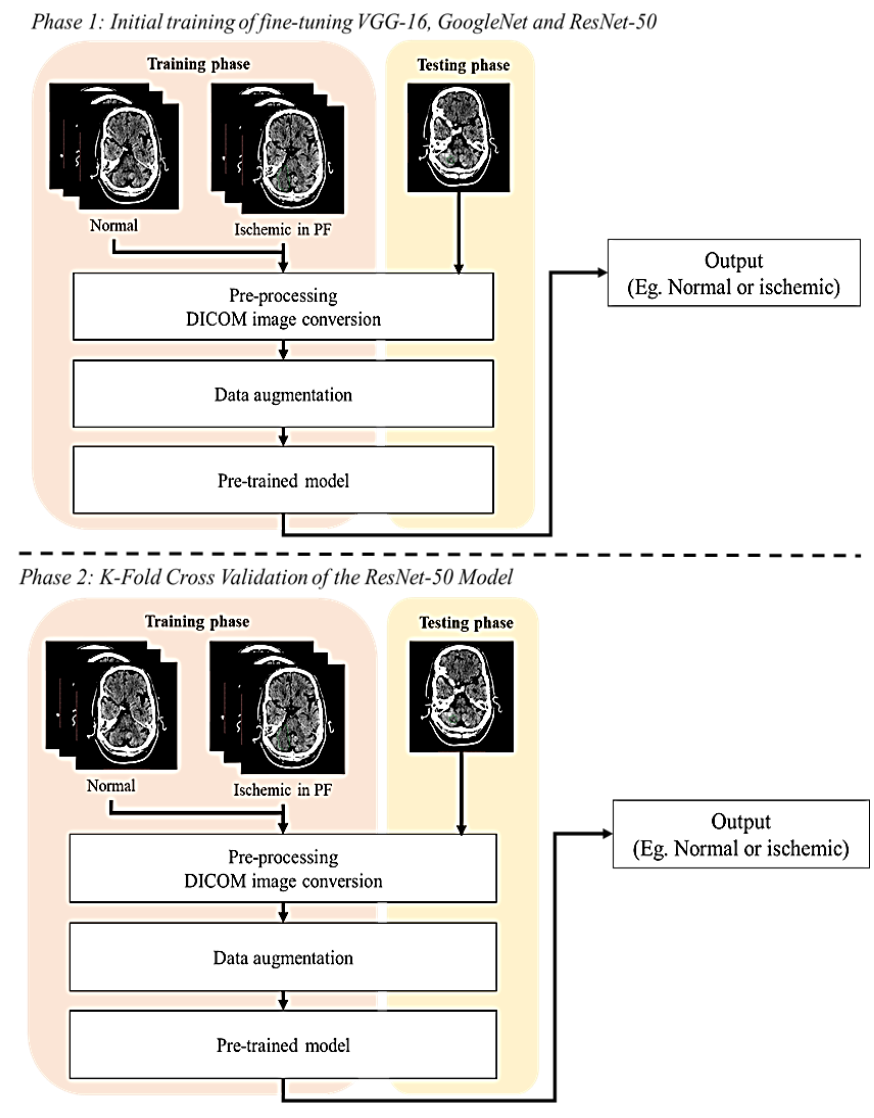

Fig. 5. The Overall Proposed Workflow.

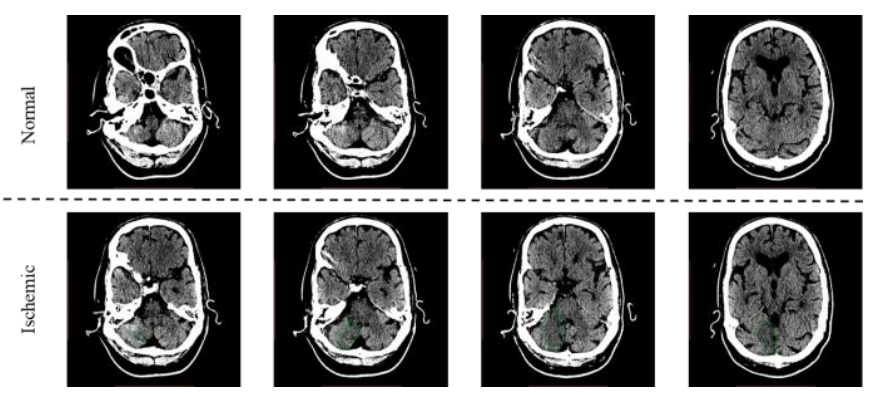

Fig. 6. Samples of Normal and Ischemic (Marked with Green Region) in PF of Brain CT Images. 


\section{B. Pre-Processing DICOM Image Conversion}

The pre-processing operation starts with converting the 16bit of DICOM image into 8-bit of grayscale range using Equation 1 and 2 for visualisation purpose. In order to enhance the visibility of the normal and abnormal tissues, the window setting of window width $\left(W_{w}\right)$ and window center $\left(W_{c}\right)$ must be in the range of +40 to +80 Hounsefield unit (HU) [32]. It is important to note that both $W_{w}$ and $W_{c}$ of $40 \mathrm{HU}$, recommended by the radiologist provides a solution that satisfies the visibility of acute ischemic in CT image [33].

$W_{c(P V)}=\frac{W_{c(H U)}-R_{i}}{R_{S}}$

$I_{\text {out }}=\left(I_{\max }-I_{\min }\right) \times \frac{I_{\text {in }}-\left(W_{c(P V)}-\frac{W_{w}}{2}\right)}{W_{w}}$

where $I_{\text {out }}$-Output value of grayscale intensity (Output image), $I_{\text {in }}$-Input value of pixel (Input image), $I_{\max }-$ Maximum grayscale intensity value $\left(I_{\max }=255\right), I_{\min }-$ Minimum grayscale intensity value $\left(I_{\min }=0\right), W_{c(P V)}-$ Window center in $\mathrm{PV}, W_{c(H U)}$-Window center in $\mathrm{HU}$, $W_{w(H U)}$-Window width in HU, $P V$-Pixel value, $R_{i}$-Rescale intercept, $R_{S}$-Rescale slope.

\section{Data Augmentation}

Training a deep learning network for classification demands a large training image. Image augmentation usually will be applied to boost deep learning performance by creating training images in different augmentation techniques [34]. Simultaneously, this technique also helps to prevent overfitting specifically for small dataset [18]. By having a small dataset, the network sometimes is unable to adapt to the new data. Therefore, in this stage, the augmentation technique which is horizontal flipping will be applied to increase the number of training images. Each image will create one (1) random artificial image as illustrated in Fig. 7.

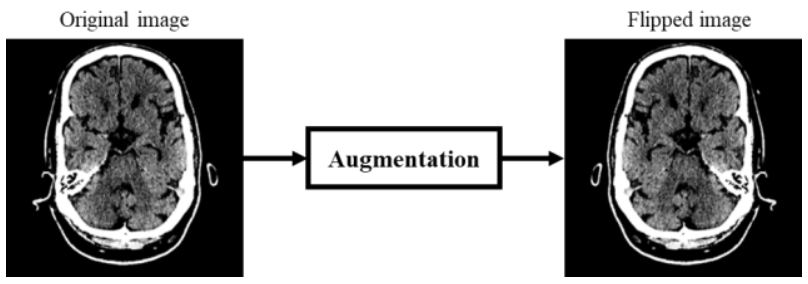

Fig. 7. Image before and after Augmentation Process.

\section{Phase 1: Initial Training of Fine-Tuning VGG-16, GoogleNet and ResNet-50}

The pre-trained models; Firstly, VGG-16, GoogleNet and ResNet-50 are trained and their respective performances are assessed based on the training and validation phase. The finetuning technique is applied in which the pre-trained models are trained and the connection weights are transferred to be adapted to the desired task. All layers with initial weights except fully connected layer are retrained without freezing. The dataset is divided into $80-20 \%$ for training and testing respectively. The CT images also are re-sampled to $224 \times 224$ pixels resolutions to match the input requirement of deep transfer learning models. Previous CNN pre-trained models are trained with input channels of red, green and blue. In this experiment, the input layer of $224 \times 224 \times 3$ is replicated with a new layer of $224 \times 224 \times 1$ since the image is in a grayscale form. Subsequently, the final layers of the pre-trained models are replaced with new layers. Fine tune the deeper layer is important for the models to learn the specific features based on the new dataset and classes. Throughout the training, Adam optimizer has been implemented to reduce loss function. The initial learning rate is set to $1 \mathrm{e}^{-4}$ and the model is trained for 3 epochs with a batch size of 20 and Squared Gradient Decay Factor (SGDF) of 0.99. After the training, the best pre-trained model is selected based on performance metrics for the validation set.

\section{E. Phase 2: K Fold Cross Validation of the ResNet-50 Model}

In phase 1, ResNet-50 model has achieved the best performance. Further details of the results can be found in Section IV (A). The best predictive model will be properly assessed through 5-fold cross validation in phase 2 to verify that model biasing and generalization errors are counteracted. This type of validation will evaluate the trained model using each of the four partitions and the remaining one is under validation. Thus, the ResNet-50 model is trained five times and the average accuracy of 5-fold cross validation is computed.

\section{EXPERIMENTAL RESULTS AND DISCUSSION}

In this section, a detailed analysis of experimental setup results is discussed. The performance of predictive models have been evaluated through traditional metrics performance; precision, recall, F1-score, specificity, accuracy and processing speed. The traditional metrics to assess the performance of the proposed method are derived based on true positive (TP), true negative (TN), false positive (FP) and false negative (FN) concept as described in Table I. Meanwhile, $k$-fold cross validation is employed to verify the performance of the selected model; ResNet-50 in classifying the image as either normal or abnormal (ischemic).

TABLE. I. ASSESSMENT METRICS FOR ClASSIFIER PERFORMANCE

\begin{tabular}{|l|c|l|}
\hline Metrics & Formula & Description \\
\hline Precision & $\frac{T P}{T P+F P}(\times 100 \%)$ & $\begin{array}{l}\text { Percentage of classified } \\
\text { slices that are actually } \\
\text { positive }\end{array}$ \\
\hline Recall & $\frac{T P}{T P+F N}(\times 100 \%)$ & $\begin{array}{l}\text { Percentage of slices } \\
\text { correctly classified }\end{array}$ \\
\hline F1-score & $\frac{2 T P}{2 T P+F P+F N}(\times 100 \%)$ & $\begin{array}{l}\text { Percentage of } \\
\text { classification } \\
\text { performance in term of } \\
\text { recall and precision }\end{array}$ \\
\hline Specificity & $\frac{T N}{F P+T N}(\times 100 \%)$ & $\begin{array}{l}\text { Percentage of } \\
\text { predicting the negative } \\
\text { slices }\end{array}$ \\
\hline Accuracy & $\frac{T P+T N}{T P+T N+F P+F N}(\times 100 \%)$ & $\begin{array}{l}\text { Percentage true or false } \\
\text { slices correctly } \\
\text { classified }\end{array}$ \\
\hline
\end{tabular}




\section{A. Phase 1: Initial Training of Fine-Tuning VGG-16, GoogleNet and ResNet-50}

The result of initial training of VGG-16, GoogleNet and ResNet-50 demonstrate average accuracy of $92.2 \%, 99.4 \%$ and $100 \%$ respectively as shown in Table II. It can be seen that the VGG-16 in stage-1 training has achieved $86.5 \%$ of precision, $92.8 \%$ of F1-score and $84.4 \%$ of specificity by misclassifying 25 images in the normal class. Meanwhile, GoogleNet in stage1 training has surpassed the performance of VGG-16 with 98.8\% of precision, $99.4 \%$ of F1-score and $98.8 \%$ of specificity by misclassifying only two images in the normal class. This model closely follows the performance of the ResNet-50 which delivers $100 \%$ for all metrics performance. Although misclassified cases have been found in a normal class, good performance has been demonstrated by these three models. All models provide $100 \%$ of sensitivity with all false negative cases are correctly classified.

Therefore, to ensure these models are properly trained, they are studied in further detail though validation phase as tabulated in Table III. The average validation accuracy of ResNet-50 is brought up to $100 \%$ as compared to VGG-16 and GoogleNet. It also can be observed that VGG-16 in phase-1 validation has yielded $83.3 \%$ of precision, $90.9 \%$ of F1-score and $80 \%$ of specificity by misclassifying eight images in the normal class. GoogleNet has made an improvement in phase-1 validation with $97.6 \%$ of precision, $98.8 \%$ of F1-score and $97.5 \%$ of specificity by misclassifying only one image in the normal class. Among these three models, ResNet-50 have provided the flexibility of being applicable in the case of classifying normal and ischemic PF slices of CT images.

The example of false predicted images in VGG-16 and GoogleNet in stage-1 are shown in Fig. 8. The predicted label shows the PF slices as abnormal while the true class label is normal. The probability for the VGG-16 to detect the PF slice as normal has achieved only 0.0819 . In the meantime, the GoogleNet identifies the PF slice as normal with a probability value of 0.4676 . These outputs are expected to be similar in colour intensity between the normal and ischemic region of the PF slices.

TABLE. II. TRAINING PERFORMANCE

\begin{tabular}{|l|l|l|l|l|l|}
\hline Models & Precision & Recall & $\begin{array}{l}\text { F1- } \\
\text { score }\end{array}$ & Specificity & Accuracy \\
\hline VGG-16 & $86.5 \%$ & $100 \%$ & $92.8 \%$ & $84.4 \%$ & $92.2 \%$ \\
\hline GoogleNet & $98.8 \%$ & $100 \%$ & $99.4 \%$ & $98.8 \%$ & $99.4 \%$ \\
\hline ResNet-50 & $100 \%$ & $100 \%$ & $100 \%$ & $100 \%$ & $100 \%$ \\
\hline
\end{tabular}

TABLE. III. VALIDATION PERFORMANCE

\begin{tabular}{|l|l|l|l|l|l|}
\hline Models & Precision & Recall & $\begin{array}{l}\text { F1- } \\
\text { score }\end{array}$ & Specificity & Accuracy \\
\hline VGG-16 & $83.3 \%$ & $100 \%$ & $90.9 \%$ & $80 \%$ & $90 \%$ \\
\hline GoogleNet & $97.6 \%$ & $100 \%$ & $98.8 \%$ & $97.5 \%$ & $98.8 \%$ \\
\hline ResNet-50 & $100 \%$ & $100 \%$ & $100 \%$ & $100 \%$ & $100 \%$ \\
\hline
\end{tabular}

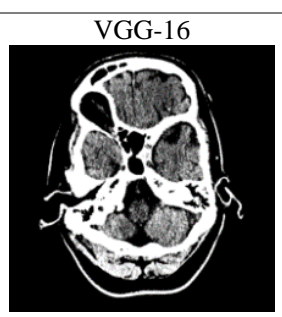

Predicted: Abnormal Actual: Normal Probability: 0.0819

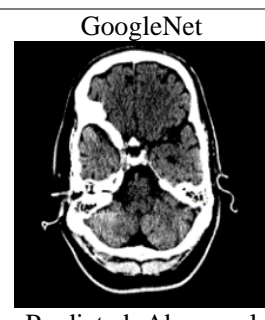

Predicted: Abnormal

Actual: Normal

Probability: 0.4676
Fig. 8. Incorrect Classified Images after Validation.

\section{B. Phase 2: K-Fold Cross Validation of the ResNet-50 Model}

The best model in the phase-1 is retrained using 5-fold cross validation as discussed in Section III (E). Table IV shows the performance results obtained for all the 5-fold cross validation. Similar to the phase-1, the model's performance performs $100 \%$ for validation average precision, recall F1score, specificity and accuracy for normal and ischemic classes. It can be observed that the ResNet-50 model correctly classified and assigned the categories of the images for all folds. The visual representations learned by the ResNet-50 model seems well suited to match the visualization. Fig. 9 demonstrates the feature map of a sample image in various layers of ResNet-50 model. The first layer differs significantly from the other layers as it performs as a group of different edge detectors and almost all the information is preserved by the activations as demonstrated in Fig. 9(b). The model described here may, therefore, keep less information for higher layers and somewhat more abstract as depicted in Fig. 9(c). Further layer in Fig. 9(d) represent the non-existing encoded pattern of the blank filter in the input image.

TABLE. IV. The Average Precision, ReCAll, F1-Score, SPecificity AND VALIDATION ACCURACY

\begin{tabular}{|l|l|l|l|l|l|}
\hline Classes & $\begin{array}{l}\text { Precision } \\
(\boldsymbol{\%})\end{array}$ & $\begin{array}{l}\text { Recall } \\
(\boldsymbol{\%})\end{array}$ & $\begin{array}{l}\text { F1- } \\
\text { score } \\
(\boldsymbol{\%})\end{array}$ & $\begin{array}{l}\text { Specificity } \\
(\boldsymbol{\%})\end{array}$ & $\begin{array}{l}\text { Accuracy } \\
(\boldsymbol{\%})\end{array}$ \\
\hline Normal & 100 & 100 & 100 & 100 & 100 \\
\hline Ischemic & 100 & 100 & 100 & 100 & \\
\hline
\end{tabular}
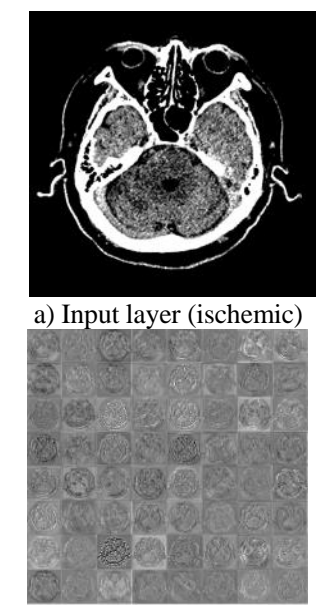

c) Feature maps at layer 70

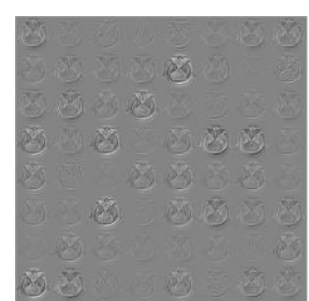

b) Feature maps at layer 2

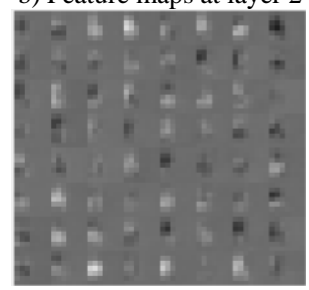

d) Feature maps at layer 170
Fig. 9. Feature Map of Sample Image at different Layers. 


\section{Processing Speed}

The processing speed of the pre-trained models also is evaluated for in training and validation of the PF CT images as tabulated in Table V. Although VGG-16 consists of the least number of layers, poor performance of processing speed has been demonstrated for training and validation. As shown in the results, VGG-16 takes a longer processing speed to train, compared with 6 minutes and 15 minutes training time of GoogleNet and ResNet-50 respectively. In order to improve the accuracy performance of VGG-16, this model requires more epoch which demands a longer processing speed in training. GoogleNet appears to possess relatively good processing speed in comparison to other proposed pre-trained models. The processing speed of ResNet-50 for training and validation demonstrates that the model seems comparable that in GoogleNet model. ResNet-50 only takes around 0.19 seconds on average to classify an image. The ResNet-50 model also requires less epoch to achieve $100 \%$ accuracy. This highprocessing speed of proposed model may offer some benefits in practical usage in hospitals to assist radiologists in their daily tasks.

\section{Comparison to other Methods}

There are several studies on DL that have been conducted to address the classification of normal and ischemic brain CT images on the anterior part using different database. Our study is conducted specifically on PF slices. Table VI shows the comparison of the related works on automated classification of the normal and ischemic image using CT image. Pereira et al. [19] use the architecture of CIFAR-10 and ImageNet with the integration of PSO for the classification of normal and ischemic images. They perform their proposed method on 300 CT brain images and have achieved $99 \%$ accuracy. Chin et al. [20] develop a CNN model from scratch for the patches classification purpose. They have reported accuracy performance with more than $90 \%$ for 256 CT images. Dourado et al. [12] compare the performance of CNN models as the feature extraction with different ML classifiers. They achieved $100 \%$ accuracy with $840 \mathrm{CT}$ images. In the above-mentioned state-of-the-art studies, there are two studies which used CNN as feature extraction and classifier. Meanwhile, there is only one study that applies CNN and ML as feature extraction and classifier respectively. In our proposed study, three CNN models are applied and the models performance to classify the normal and ischemic in PF CT slices is observed. The proposed method has achieved $100 \%$ accuracy with 5-fold cross validation using 400 images with ResNet-50 model.

The great advantage of the proposed method is that this is the first time the CNN pre-trained-based approach is investigated to use in classifying normal and ischemic mainly in PF slices. The application of pre-trained model as a deep transfer learning offers benefit to address the poor performance of DL using a limited amount of data. Gathering a sufficient amount of data can be a difficult process since there is no existing data for such amount which publicly available to be used for the DL purpose. However, this limitation can be addressed using the data augmentation technique.
TABLE. V. PROCESSING SPEEd OF TRAINING AND VALIDATION

\begin{tabular}{|l|l|l|l|}
\hline \multirow{2}{*}{ CNN models } & Training & \multicolumn{2}{|l|}{ Validation } \\
\cline { 2 - 4 } & 1 set & 1 set & 1 image \\
\hline VGG-16 & $\begin{array}{l}33 \text { minutes and 22 } \\
\text { seconds }\end{array}$ & 32.9 seconds & $\begin{array}{l}0.41 \\
\text { seconds }\end{array}$ \\
\hline GoogleNet & $\begin{array}{l}6 \text { minutes and 12 } \\
\text { seconds }\end{array}$ & 7.1 seconds & $\begin{array}{l}0.08 \\
\text { seconds }\end{array}$ \\
\hline ResNet-50 & $\begin{array}{l}15 \text { minutes and } 37 \\
\text { seconds }\end{array}$ & 15.4 seconds & $\begin{array}{l}0.19 \\
\text { seconds }\end{array}$ \\
\hline
\end{tabular}

TABLE. VI. COMPARISON OF WORKS CONDUCTED ON ISCHEMIC STROKE DETECTION USING DL

\begin{tabular}{|l|l|l|l|l|}
\hline Author(s) & Application & $\begin{array}{l}\text { Number of } \\
\text { images }\end{array}$ & $\begin{array}{l}\text { Method/architect } \\
\text { ure uses }\end{array}$ & Accuracy \\
\hline $\begin{array}{l}\text { Pereira et } \\
\text { al. [19] }\end{array}$ & $\begin{array}{l}\text { Anterior } \\
\text { slices }\end{array}$ & 300 & $\begin{array}{l}\text { CIFAR-10 + } \\
\text { ImageNet + PSO }\end{array}$ & $\begin{array}{l}\text { Up to } \\
99 \%\end{array}$ \\
\hline $\begin{array}{l}\text { Chin et } \\
\text { al. [20] }\end{array}$ & $\begin{array}{l}\text { Anterior } \\
\text { slices }\end{array}$ & 256 & CNN from scratch & $>90 \%$ \\
\hline $\begin{array}{l}\text { Dourado } \\
\text { et al.[12] }\end{array}$ & $\begin{array}{l}\text { Anterior } \\
\text { slices }\end{array}$ & 840 & $\begin{array}{l}\text { CNN + ML } \\
\text { classifiers }\end{array}$ & $100 \%$ \\
\hline $\begin{array}{l}\text { The } \\
\text { proposed } \\
\text { model }\end{array}$ & PF slices & 400 & ResNet-50 & $100 \%$ \\
\hline
\end{tabular}

\section{CONCLUSION}

In this study, the aim of this experiment is to evaluate three popular pre-trained deep learning CNN architecture; VGG-16, GoogleNet and ResNet-50 to classify normal and ischemic in $\mathrm{PF}$ slices of brain CT. In contrast to the usual study of DL in brain CT, the application of DL is studied in further detail for $\mathrm{PF}$ slices. An intensive experiment has been conducted on the CT dataset of PF using DL with augmentation technique. Among these three proposed models, ResNet-50 achieves better performance than the rest of the state-of-the-art methods with $100 \%$ accuracy for initial training, validation and 5-fold cross validation. The experiment also shows that ResNet-50 is generally applicable to the ischemic classification in PF slices with good processing speed that is less than 1 seconds. Therefore, this model can help to improve the diagnostic performance with better computational efficiency. In future studies, the integration of the detection task with classification can be developed for localizing the location of ischemic in PF slices. A Graphical User Interface (GUI) also will be developed for assisting the diagnosis process in the real-time application.

\section{ACKNOWLEDGMENT}

This study is supported by Universiti Tun Hussein Onn Malaysia and UKM Medical Centre under TIER 1 Vot H203 and GPPS Vot U954 Research Grants. 


\section{REFERENCES}

[1] Aziz ZA, Lee YY, Ngah BA, Sidek NN, Looi I, Hanip MR, Basri HB: Acute stroke registry Malaysia, 2010-2014: results from the National Neurology Registry. Journal of Stroke and Cerebrovascular Diseases. 24(12), 2701-2709 (2015).

[2] Gomez, CR: Time is brain: the stroke theory of relativity. Journal of Stroke and Cerebrovascular Diseases. 27(8), 2214-2227 (2018).

[3] Dubey P, Pandey S, Moonis G: Acute stroke imaging: recent updates. Stroke Research and Treatment. 2013, 6 pages (2013).

[4] Kooi CW, Peng HC, Aziz ZA, Looi I: A review of stroke research in Malaysia from 2000-2014. Med J Malaysia. 71, 58-69 (2016).

[5] Habibi-koolaee M, Shahmoradi L, Niakan Kalhori SR, Ghannadan H, Younesi E: Prevalence of Stroke Risk Factors and Their Distribution Based on Stroke Subtypes in Gorgan: A Retrospective Hospital-Based Study-2015-2016. Neurology Research International. (2018).

[6] El-Koussy M, Schroth G, Brekenfeld C, Arnold M: Imaging of acute ischemic stroke. European Neurology. 72(5-6), 309-16 (2014).

[7] Lin, M P.: Imaging of ischemic stroke. Continuum: Lifelong Learning in Neurology. 22(5):1399 (2016).

[8] Sharon M, Boyle K, Yeung R, Symons SP, Boulos MI, Aviv RI: The predictive value of a targeted posterior fossa multimodal stroke protocol for the diagnosis of acute posterior ischemic stroke. Neurovascular Imaging. 2(1):3 (2016).

[9] Hixson HR, Leiva-Salinas C, Sumer S, Patrie J, Xin W, Wintermark M: Utilizing dual energy CT to improve CT diagnosis of posterior fossa ischemia. Journal of Neuroradiology. 43(5), 346-352 (2016).

[10] Hwang DY, Silva GS, Furie KL, Greer DM: Comparative sensitivity of computed tomography vs. magnetic resonance imaging for detecting acute posterior fossa infarct. The Journal of emergency medicine. 42(5), 559-65 (2016).

[11] Tang FH, Ng DK, Chow DH: An image feature approach for computeraided detection of ischemic stroke. Computers in biology and medicine. 41(7), 529-36 (2011).

[12] Dourado Jr CM, da Silva SP, da Nóbrega RV, Barros AC, Rebouças Filho PP, de Albuquerque VH: Deep learning IoT system for online stroke detection in skull computed tomography images. Computer Networks. 152, 25-39 (2019).

[13] Tyan YS, Wu MC, Chin CL, Kuo YL, Lee MS, Chang HY: Ischemic stroke detection system with a computer-aided diagnostic ability using an unsupervised feature perception enhancement method. Journal of Biomedical Imaging. 2014, 19 (2014).

[14] Kanchana R, Menaka R: Computer reinforced analysis for ischemic stroke recognition: a review. Indian J. Sci. Technol. 8(35), 81006 (2015).

[15] Kanchana R, Menaka R: A novel approach for characterisation of ischaemic stroke lesion using histogram bin-based segmentation and gray level co-occurrence matrix features. The Imaging Science Journal. 65(2), 124-36 (2017).

[16] Aggarwal N, Agrawal RK: First and second order statistics features for classification of magnetic resonance brain images. Journal of Signal and Information Processing. 3(02), 146 (2012).

[17] Suzuki, K.: Overview of deep learning in medical imaging. Radiological physics and technology. 10(3), 257-73 (2017).
[18] Talo M, Baloglu UB, Yıldırım Ö, Acharya UR: Application of deep transfer learning for automated brain abnormality classification using MR images. Cognitive Systems Research. 54, 176-88 (2019).

[19] Pereira DR, Reboucas Filho PP, de Rosa GH, Papa JP, de Albuquerque VH: Stroke lesion detection using convolutional neural networks. In 2018 International joint conference on neural networks (IJCNN), pp. 16. IEEE (2018).

[20] Chin CL, Lin BJ, Wu GR, Weng TC, Yang CS, Su RC, Pan YJ: An automated early ischemic stroke detection system using CNN deep learning algorithm. In 2017 IEEE 8th International Conference on Awareness Science and Technology (iCAST), pp. 368-372. IEEE (2017).

[21] Tajbakhsh N, Shin JY, Gurudu SR, Hurst RT, Kendall CB, Gotway MB, Liang J: Convolutional neural networks for medical image analysis: Full training or fine tuning?. IEEE transactions on medical imaging. 35(5), 1299-312 (2016).

[22] Pak M, Kim S: A review of deep learning in image recognition. In 2017 4th international conference on computer applications and information processing technology (CAIPT), pp. 1-3. IEEE (2017).

[23] He K, Zhang X, Ren S, Sun J: Deep residual learning for image recognition. In Proceedings of the IEEE conference on computer vision and pattern recognition, pp. 770-778. IEEE (2016).

[24] Szegedy C, Liu W, Jia Y, Sermanet P, Reed S, Anguelov D, Erhan D, Vanhoucke V, Rabinovich A: Going deeper with convolutions. In Proceedings of the IEEE conference on computer vision and pattern recognition, pp. 1-9. IEEE (2015).

[25] Simonyan K, Zisserman A. Very deep convolutional networks for largescale image recognition. arXiv preprint arXiv:1409.1556. (2014).

[26] Kamal, H.: Machine learning in acute ischemic stroke neuroimaging. Frontiers in neurology. 9, 945 (2018).

[27] Nouh A, Remke J, Ruland S: Ischemic posterior circulation stroke: a review of anatomy, clinical presentations, diagnosis, and current management. Frontiers in neurology. 5, 30 (2014).

[28] Tsochatzidis L, Costaridou L, Pratikakis I: Deep Learning for Breast Cancer Diagnosis from Mammograms-A Comparative Study. Journal of Imaging. 5(3), 37 (2019).

[29] Khan S, Yairi T: A review on the application of deep learning in system health management. Mechanical Systems and Signal Processing. 107, 241-65 (2018).

[30] Agarwal R, Diaz O, Lladó X, Yap MH, Martí R: Automatic mass detection in mammograms using deep convolutional neural networks. Journal of Medical Imaging. 6(3), 031409 (2019).

[31] Ji Q, Huang J, He W, Sun Y: Optimized Deep Convolutional Neural Networks for Identification of Macular Diseases from Optical Coherence Tomography Images. Algorithms. 12(3), 51 (2019).

[32] Ee CS, Sim KS, Teh V, Ting FF: Estimation of window width setting for CT scan brain images using mean of greyscale level to standard deviation ratio. In 2016 International Conference on Robotics, Automation and Sciences (ICORAS), pp. 1-6. IEEE (2016).

[33] Suberi AA, Zakaria WN, Tomari R, Fuad NF: Classification of Posterior Fossa CT Brain Slices using Artificial Neural Network. Procedia Computer Science. 135, 170-177 (2018).

[34] Lopez AR, Giro-i-Nieto X, Burdick J, Marques O: Skin lesion classification from dermoscopic images using deep learning techniques. In 2017 13th IASTED International Conference on Biomedical Engineering (BioMed), pp. 49-54. IEEE (2017). 